\title{
THE FINITE DIFFERENCE METHOD FOR TRANSIENT CONVECTION-DIFFUSION PROBLEMS
}

\author{
Ewa Majchrzak ${ }^{1,2}$, Lukasz Turchan ${ }^{1}$ \\ ${ }^{I}$ Department of Strength of Materials and Computational Mechanics \\ Silesian University of Technology, Poland \\ ${ }^{2}$ Institute of Mathematics, Czestochowa University of Technology, Poland \\ ewa.majchrzak@polsl.pl,lukasz.turchan@polsl.pl
}

\begin{abstract}
The convection-diffusion equation (1D problem) is considered. At first, the unknown temperature $T$ is expanded into a Taylor series with respect to time taking into account its three components. Next, using the convection-diffusion equation and equation obtained from the differentiation of this equation, the way of temperature $T$ computations is shown. In this new equation the high order derivatives with respect to spatial co-ordinate appear and the approximation of these derivatives is also discussed. The explicit scheme is used and the stability criteria are formulated. Finally, the results of computations are shown.
\end{abstract}

\section{Introduction}

The following convection-diffusion equation is considered [1-4]

$$
0<x<L: \quad c \rho\left[\frac{\partial T(x, t)}{\partial t}+\varepsilon u \frac{\partial T(x, t)}{\partial x}\right]=\lambda \frac{\partial^{2} T(x, t)}{\partial x^{2}}+Q(x, t)
$$

where $c$ is the specific heat, $\rho$ is the mass density, $\lambda$ is the thermal conductivity, $\varepsilon$ denotes the porosity (the ratio of liquid volume to the total volume), $u$ is the velocity, $T$ denotes the temperature, $t$ is a time, $Q(x, t)$ is the capacity of internal heat sources. All thermophysical parameters in equation (1) are assumed to be known and constant. The equation (1) can be written in the form

$$
\frac{\partial T}{\partial t}=a \frac{\partial^{2} T}{\partial x^{2}}-\varepsilon u \frac{\partial T}{\partial x}+\frac{Q}{c \rho}
$$

where $a=\lambda /(c \rho)$ is the diffusion coefficient.

The difficulties connected with the proper solution of convection-diffusion equation (2) appear when the second term on the right-hand side (convection) is dominated [5]. 
Using the explicit scheme of the finite difference method [6-10] the following approximation of equation (2) for the internal node $i$ can be proposed

$$
\frac{T_{i}^{f}-T_{i}^{f-1}}{\Delta t}=a \frac{T_{i-1}^{f-1}-2 T_{i}^{f-1}+T_{i+1}^{f-1}}{h^{2}}-\varepsilon u \frac{T_{i+1}^{f-1}-T_{i-1}^{f-1}}{2 h}+\frac{Q_{i}^{f-1}}{c \rho}
$$

where $\Delta t=t^{f}-t^{f-1}$ is the time step and $h$ is the mesh step, respectively.

From equation (3) results that

$$
T_{i}^{f}=\left(1-\frac{2 a \Delta t}{h^{2}}\right) T_{i}^{f-1}+\left(\frac{a \Delta t}{h^{2}}+\frac{\varepsilon u \Delta t}{2 h}\right) T_{i-1}^{f-1}+\left(\frac{a \Delta t}{h^{2}}-\frac{\varepsilon u \Delta t}{2 h}\right) T_{i+1}^{f-1}+\frac{Q_{i}^{f-1}}{c \rho} \Delta t
$$

The criteria of stability are the following

$$
1-\frac{2 a \Delta t}{h^{2}} \geq 0, \quad \frac{a}{h^{2}}-\frac{\varepsilon u}{2 h} \geq 0 \rightarrow h \leq \frac{2 a}{\varepsilon u}, \quad \Delta t \leq \frac{h^{2}}{2 a}
$$

In the paper the other algorithm based on the FDM is proposed. At first, the function $T$ is expanded into a Taylor series with respect to time taking into account its three components. Next, using the equation (2) and equation obtained from the differentiation of this equation with respect to time, the method of temperature $T^{f}$ computations is shown. In this new equation the high order derivatives with respect to $x$ appear and the approximation of these derivatives is also discussed.

\section{Convection-diffusion equation - discretization with respect to time}

Function $T$ is expanded into a Taylor series taking into account its three components [5]

$$
T^{f}=T^{f-1}+\Delta t \frac{\partial T^{f-1}}{\partial t}+\frac{(\Delta t)^{2}}{2} \frac{\partial^{2} T^{f-1}}{\partial t^{2}}
$$

From equation (6) one has

$$
\frac{T^{f}-T^{f-1}}{\Delta t}=\frac{\partial T^{f-1}}{\partial t}+\frac{\Delta t}{2} \frac{\partial^{2} T^{f-1}}{\partial t^{2}}
$$

Equation (2) is differentiated with respect to time

$$
\frac{\partial^{2} T}{\partial t^{2}}=a \frac{\partial^{2}}{\partial x^{2}}\left(\frac{\partial T}{\partial t}\right)-\varepsilon u \frac{\partial}{\partial x}\left(\frac{\partial T}{\partial t}\right)+\frac{1}{c \rho} \frac{\partial Q}{\partial t}
$$


The equations (2), (8) can be written for time $t^{f-1}$

$$
\frac{\partial T^{f-1}}{\partial t}=a \frac{\partial^{2} T^{f-1}}{\partial x^{2}}-\varepsilon u \frac{\partial T^{f-1}}{\partial x}+\frac{Q}{c \rho}
$$

and

$$
\frac{\partial^{2} T^{f-1}}{\partial t^{2}}=a \frac{\partial^{2}}{\partial x^{2}}\left(\frac{\partial T^{f-1}}{\partial t}\right)-\varepsilon u \frac{\partial}{\partial x}\left(\frac{\partial T^{f-1}}{\partial t}\right)+\frac{1}{c \rho} \frac{\partial Q}{\partial t}
$$

Introducing (9) and (10) into (7) one has

$$
\begin{aligned}
& \frac{T^{f}-T^{f-1}}{\Delta t}=a \frac{\partial^{2} T^{f-1}}{\partial x^{2}}-\varepsilon u \frac{\partial T^{f-1}}{\partial x}+\frac{Q}{c \rho}+ \\
& \frac{\Delta t}{2}\left(a \frac{\partial^{2}}{\partial x^{2}}\left(\frac{\partial T^{f-1}}{\partial t}\right)-\varepsilon u \frac{\partial}{\partial x}\left(\frac{\partial T^{f-1}}{\partial t}\right)+\frac{1}{c \rho} \frac{\partial Q}{\partial t}\right)
\end{aligned}
$$

Taking into account the dependence (9) one obtains

$$
\begin{aligned}
& \frac{T^{f}-T^{f-1}}{\Delta t}=a \frac{\partial^{2} T^{f-1}}{\partial x^{2}}+\frac{\Delta t}{2}\left[a \frac{\partial^{2}}{\partial x^{2}}\left(a \frac{\partial^{2} T^{f-1}}{\partial x^{2}}-\varepsilon u \frac{\partial T^{f-1}}{\partial x}+\frac{Q}{c \rho}\right)-\right. \\
& \left.-\varepsilon u \frac{\partial}{\partial x}\left(a \frac{\partial^{2} T^{f-1}}{\partial x^{2}}-\varepsilon u \frac{\partial T^{f-1}}{\partial x}+\frac{Q}{c \rho}\right)+\frac{1}{c \rho} \frac{\partial Q}{\partial t}\right]-\varepsilon u \frac{\partial T^{f-1}}{\partial x}+\frac{Q}{c \rho}
\end{aligned}
$$

or

$$
\begin{aligned}
& \frac{T^{f}-T^{f-1}}{\Delta t}=\frac{a^{2} \Delta t}{2} \frac{\partial^{4} T^{f-1}}{\partial x^{4}}-a \varepsilon u \Delta t \frac{\partial^{3} T^{f-1}}{\partial x^{3}}+a \frac{\partial^{2} T^{f-1}}{\partial x^{2}}+ \\
& +\frac{\varepsilon^{2} u^{2} \Delta t}{2} \frac{\partial^{2} T^{f-1}}{\partial x^{2}}-\varepsilon u \frac{\partial T^{f-1}}{\partial x}+\frac{a \Delta t}{2 c \rho} \frac{\partial^{2} Q}{\partial x^{2}}-\frac{\varepsilon u \Delta t}{2 c \rho} \frac{\partial Q}{\partial x}+\frac{\Delta t}{2 c \rho} \frac{\partial Q}{\partial t}+\frac{Q}{c \rho}
\end{aligned}
$$

From equation (13) results that

$$
\begin{aligned}
& T^{f}=\frac{(a \Delta t)^{2}}{2} \frac{\partial^{4} T^{f-1}}{\partial x^{4}}-a \varepsilon u(\Delta t)^{2} \frac{\partial^{3} T^{f-1}}{\partial x^{3}}+\left(a \Delta t+\frac{(\varepsilon u \Delta t)^{2}}{2}\right) \frac{\partial^{2} T^{f-1}}{\partial x^{2}}- \\
& -\varepsilon u \Delta t \frac{\partial T^{f-1}}{\partial x}+T^{f-1}+\frac{a(\Delta t)^{2}}{2 c \rho} \frac{\partial^{2} Q}{\partial x^{2}}-\frac{\varepsilon u(\Delta t)^{2}}{2 c \rho} \frac{\partial Q}{\partial x}+\frac{(\Delta t)^{2}}{2 c \rho} \frac{\partial Q}{\partial t}+\frac{\Delta t Q}{c \rho}
\end{aligned}
$$




\section{Approximation of high order derivatives}

The next step of the algorithm presented consists in the approximation of derivatives appearing on the right-hand side of equation (14). Let us assume that $i=0,1, \ldots, n$, where $i=0$ and $i=n$ correspond to the boundary nodes, while $i=1,2, \ldots, n-1$ are the internal nodes. For the nodes $i=2,3, \ldots, n-2$ the following approximations are used [2]

$$
\begin{gathered}
\left(\frac{\partial T^{f-1}}{\partial x}\right)_{i}=\frac{T_{i+1}^{f-1}-T_{i-1}^{f-1}}{2 h} \\
\left(\frac{\partial^{2} T^{f-1}}{\partial x^{2}}\right)_{i}=\frac{T_{i+1}^{f-1}-2 T_{i}^{f-1}+T_{i-1}^{f-1}}{h^{2}} \\
\left(\frac{\partial^{3} T^{f-1}}{\partial x^{3}}\right)_{i}=\frac{T_{i+2}^{f-1}-2 T_{i+1}^{f-1}+2 T_{i-1}^{f-1}-T_{i-2}^{f-1}}{2 h^{3}} \\
\left(\frac{\partial^{4} T^{f-1}}{\partial x^{4}}\right)_{i}=\frac{T_{i+2}^{f-1}-4 T_{i+1}^{f-1}+6 T_{i}^{f-1}-4 T_{i-1}^{f-1}+T_{i-2}^{f-1}}{h^{4}}
\end{gathered}
$$

Introducing the dependences (15)-(18) into equation (14) one has

$$
\begin{aligned}
& T_{i}^{f}=\frac{3(a \Delta t)^{2}-2 a h^{2} \Delta t-(\varepsilon u h \Delta t)^{2}+h^{4}}{h^{4}} T_{i}^{f-1} \\
& +\frac{(a \Delta t)^{2}-a \varepsilon u h(\Delta t)^{2}}{2 h^{4}} T_{i+2}^{f-1}+\frac{(a \Delta t)^{2}+a \varepsilon u h(\Delta t)^{2}}{2 h^{4}} T_{i-2}^{f-1} \\
& +\frac{-4(a \Delta t)^{2}+2 a \varepsilon u h(\Delta t)^{2}+2 a h^{2} \Delta t+(\varepsilon u h \Delta t)^{2}-\varepsilon u h^{3} \Delta t}{2 h^{4}} T_{i+1}^{f-1} \\
& +\frac{-4(a \Delta t)^{2}-2 a \varepsilon u h(\Delta t)^{2}+2 a h^{2} \Delta t+(\varepsilon u h \Delta t)^{2}+\varepsilon u h^{3} \Delta t}{2 h^{4}} T_{i+1}^{f-1} \\
& +\frac{a(\Delta t)^{2}}{2 h^{2} c \rho}\left(Q_{i+1}^{f-1}-2 Q_{i}^{f-1}+Q_{i-1}^{f-1}\right)-\frac{\varepsilon u(\Delta t)^{2}}{4 h c \rho}\left(Q_{i+1}^{f-1}-Q_{i-1}^{f-1}\right) \\
& +\frac{\Delta t}{2 c \rho}\left(Q_{i}^{f}+Q_{i}^{f-1}\right)
\end{aligned}
$$

For stability conditions, the coefficients on the right-hand side terms must be positive, this means 


$$
\left\{\begin{array}{l}
3(a \Delta t)^{2}-2 a h^{2} \Delta t-(\varepsilon u h \Delta t)^{2}+h^{4} \geq 0 \\
(a \Delta t)^{2}-a \varepsilon u h(\Delta t)^{2} \geq 0 \\
-4(a \Delta t)^{2}+2 a \varepsilon u h(\Delta t)^{2}+2 a h^{2} \Delta t+(\varepsilon u h \Delta t)^{2}-\varepsilon u h^{3} \Delta t \geq 0 \\
-4(a \Delta t)^{2}-2 a \varepsilon u h(\Delta t)^{2}+2 a h^{2} \Delta t+(\varepsilon u h \Delta t)^{2}+\varepsilon u h^{3} \Delta t \geq 0
\end{array}\right.
$$

From this system of inequalities results that

$$
\begin{aligned}
& h \leq \frac{a}{\varepsilon u} \\
& \Delta t \leq \frac{2 a h^{2}}{4 a^{2}-\varepsilon^{2} u^{2} h^{2}}
\end{aligned}
$$

So, according to the conditions (21), at first the mesh step $h$ should be determined and next the proper time step $\Delta t$ should be assumed.

It should be pointed out that for the node $i=1$ the following approximations are applied

$$
\begin{gathered}
\left(\frac{\partial T^{f-1}}{\partial x}\right)_{i}=\frac{T_{i+1}^{f-1}-T_{i}^{f-1}}{h} \\
\left(\frac{\partial^{2} T^{f-1}}{\partial x^{2}}\right)_{i}=\frac{T_{i}^{f-1}-2 T_{i+1}^{f-1}+T_{i+2}^{f-1}}{h^{2}} \\
\left(\frac{\partial^{3} T^{f-1}}{\partial x^{3}}\right)_{i}=\frac{-T_{i-1}^{f-1}+3 T_{i}^{f-1}-3 T_{i+1}^{f-1}+T_{i+2}^{f-1}}{h^{3}} \\
\left(\frac{\partial^{4} T^{f-1}}{\partial x^{4}}\right)_{i}=\frac{T_{i-1}^{f-1}-4 T_{i}^{f-1}+6 T_{i+1}^{f-1}-4 T_{i+2}^{f-1}+T_{i+3}^{f-1}}{h^{4}}
\end{gathered}
$$

while for the node $i=n-1$ :

$$
\begin{gathered}
\left(\frac{\partial T^{f-1}}{\partial x}\right)_{i}=\frac{T_{i}^{f-1}-T_{i-1}^{f-1}}{h} \\
\left(\frac{\partial^{2} T^{f-1}}{\partial x^{2}}\right)_{i}=\frac{T_{i-2}^{f-1}-2 T_{i-1}^{f-1}+T_{i}^{f-1}}{h^{2}}
\end{gathered}
$$




$$
\begin{gathered}
\left(\frac{\partial^{3} T^{f-1}}{\partial x^{3}}\right)_{i}=\frac{-T_{i-2}^{f-1}+3 T_{i-1}^{f-1}-3 T_{i}^{f-1}+T_{i+1}^{f-1}}{h^{3}} \\
\left(\frac{\partial^{4} T^{f-1}}{\partial x^{4}}\right)_{i}=\frac{T_{i-3}^{f-1}-4 T_{i-2}^{f-1}+6 T_{i-1}^{f-1}-4 T_{i}^{f-1}+T_{i+1}^{f-1}}{h^{4}}
\end{gathered}
$$

The way of boundary conditions approximation is the same as in the classical finite difference method $[6,7]$.

\section{Results of computations}

The layer of thickness $L=0.05 \mathrm{~m}$ is considered. The following input data are introduced: thermal conductivity $\lambda=0.5 \mathrm{~W} /(\mathrm{mK})$, specific heat $c=4000 \mathrm{~J} /(\mathrm{kgK})$, density $\rho=1000 \mathrm{~kg} / \mathrm{m}^{3}$. For $x=0$ and $x=L$ the zero heat fluxes are assumed. The initial temperature is equal to $30^{\circ} \mathrm{C}$. The source function is of the form

$$
Q(x, t)= \begin{cases}10^{6} \mathrm{~W} / \mathrm{m}^{3}, & x \in\left[\begin{array}{ll}
0.02 \mathrm{~m}, & 0.03 \mathrm{~m}
\end{array}\right], \quad t \leq 10 \mathrm{~s} \\
0, & x \in\left[\begin{array}{ll}
0, & 0.02 \mathrm{~m}) \cup(0.03 \mathrm{~m}, \quad 0.05 \mathrm{~m}
\end{array}\right], \quad t>10 \mathrm{~s}\end{cases}
$$

Mesh step is equal to $h=0.0001 \mathrm{~m}$, time step: $\Delta t=0.01 \mathrm{~s}$.

In Figures 1-6 the temperature distributions in the domain considered for chosen moments of times and different values of velocity $u$ and porosity $\varepsilon$ are shown.

In Figures 7 and 8 the temperature history at the central node for different values of porosity under the assumption that $u=0.01 \mathrm{~m} / \mathrm{s}$ (Fig. 7) and $u=0.03 \mathrm{~m} / \mathrm{s}$ is shown.

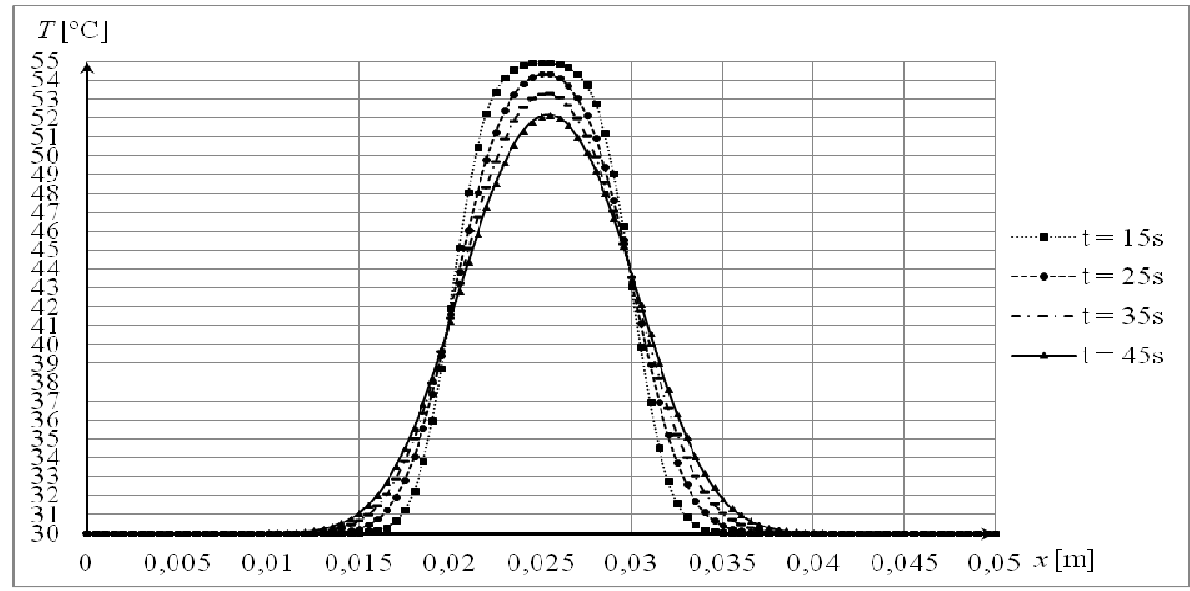

Fig. 1. Temperature distribution after $15,25,35$ and 45 seconds $(u=0.01 \mathrm{~m} / \mathrm{s}, \varepsilon=0.001)$ 


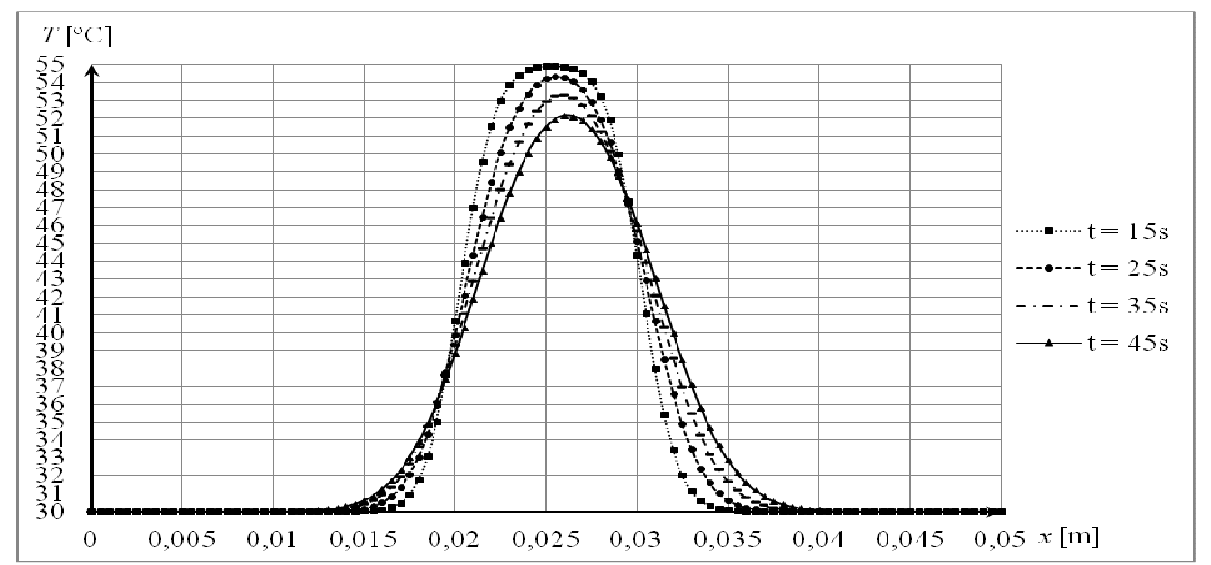

Fig. 2. Temperature distribution after $15,25,35$ and 45 seconds $(u=0.03 \mathrm{~m} / \mathrm{s}, \varepsilon=0.001)$

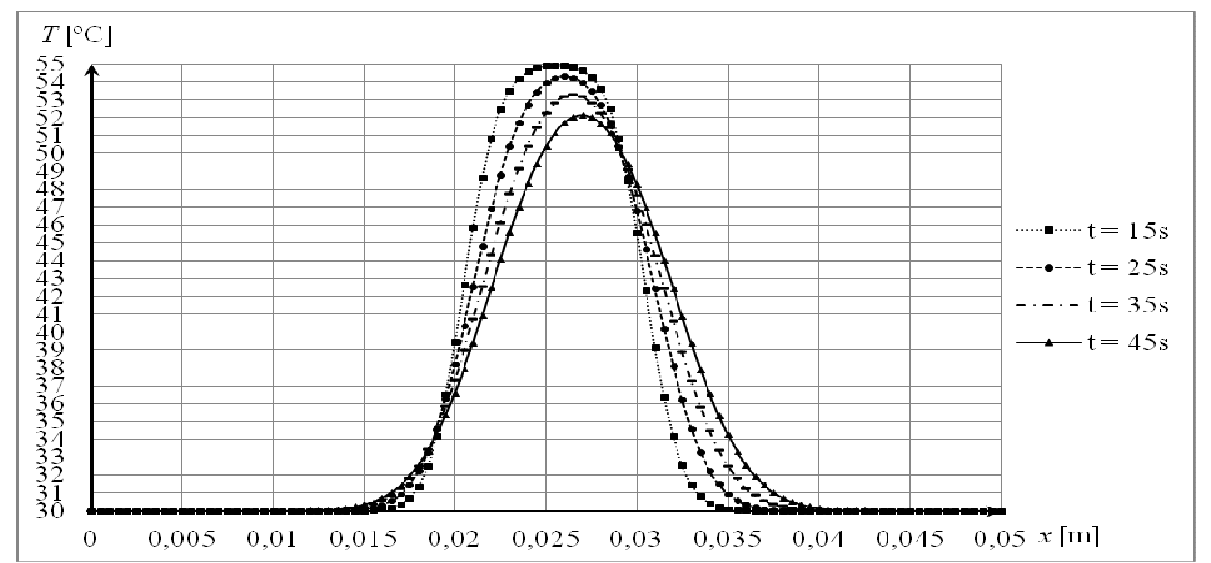

Fig. 3. Temperature distribution after $15,25,35$ and 45 seconds $(u=0.01 \mathrm{~m} / \mathrm{s}, \varepsilon=0.005)$

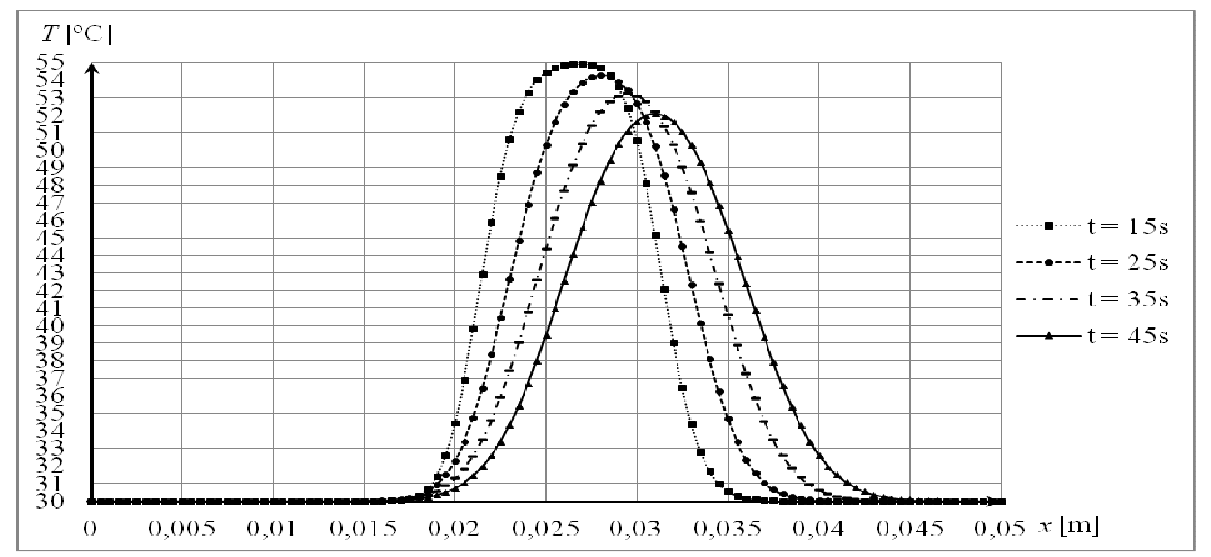

Fig. 4. Temperature distribution after $15,25,35$ and 45 seconds $(u=0.03 \mathrm{~m} / \mathrm{s}, \varepsilon=0.005)$ 


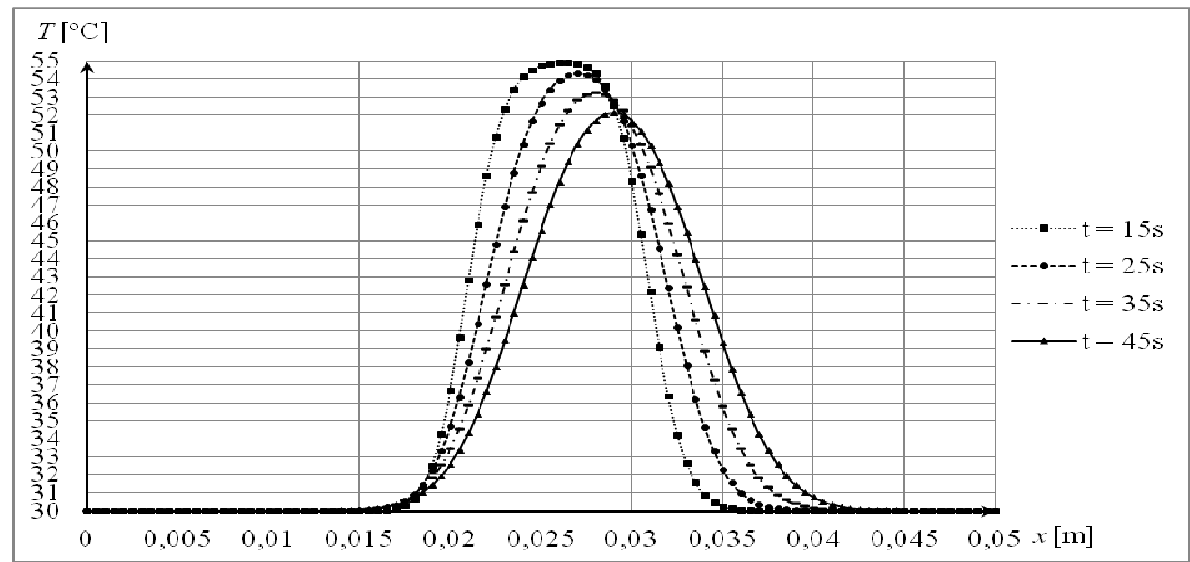

Fig. 5. Temperature distribution after $15,25,35$ and 45 seconds $(u=0.01 \mathrm{~m} / \mathrm{s}, \varepsilon=0.01)$

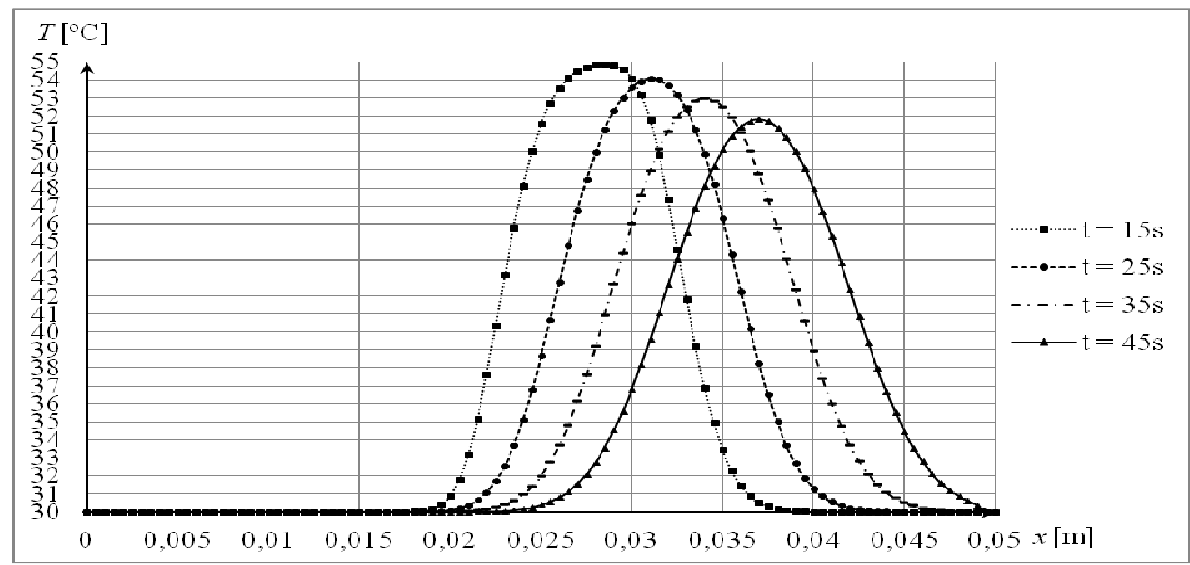

Fig. 6. Temperature distribution after $15,25,35$ and 45 seconds $(u=0.03 \mathrm{~m} / \mathrm{s}, \varepsilon=0.01)$

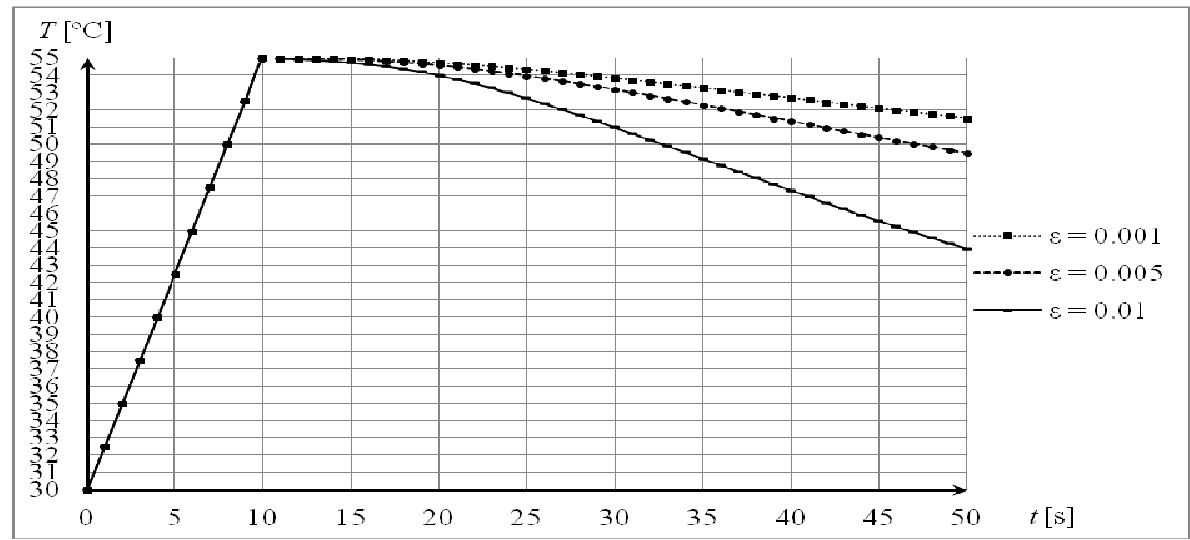

Fig. 7. Temperature history at the central node $(u=0.01 \mathrm{~m} / \mathrm{s})$ 


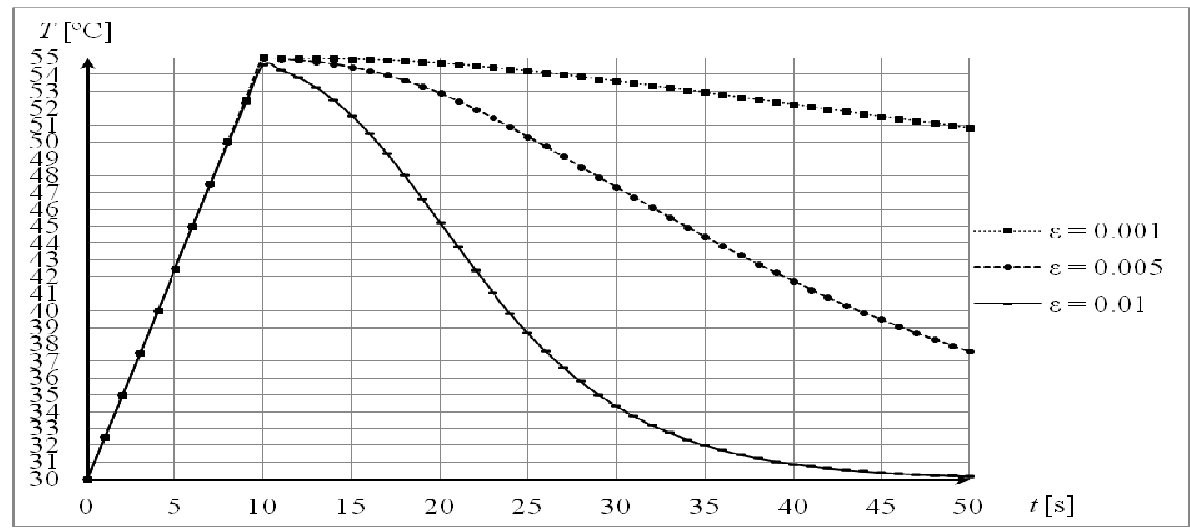

Fig. 8. Temperature history at the central node $(u=0.03 \mathrm{~m} / \mathrm{s})$

Figure 9 illustrates the temperature distribution after 25 seconds for different velocities under the assumption that porosity is equal to $\varepsilon=0.01$.

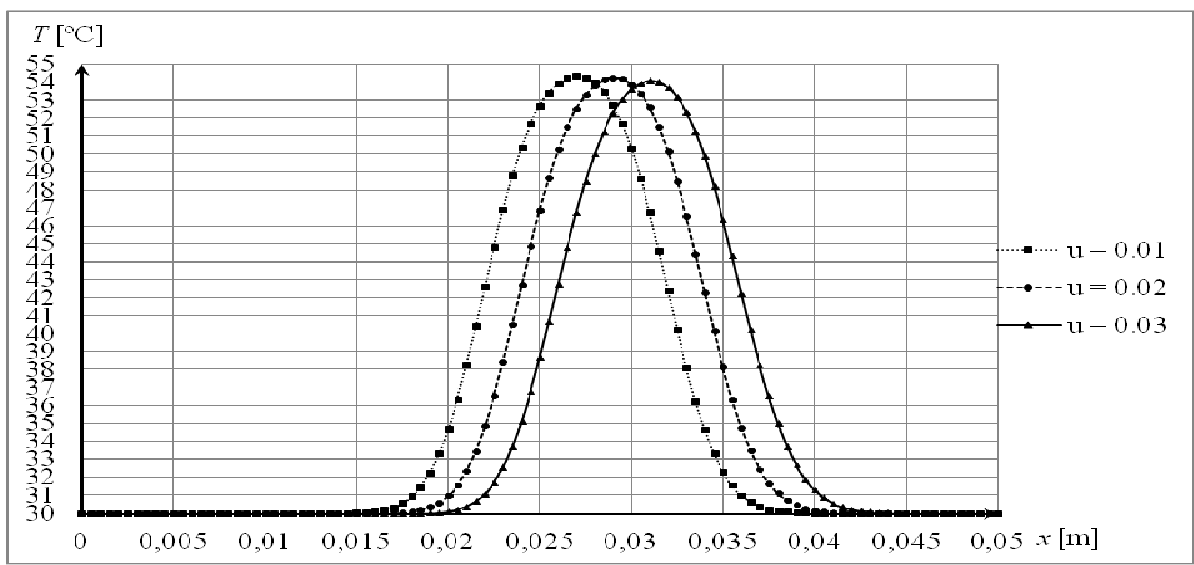

Fig. 9. Temperature distribution after $25 \mathrm{~s}(\varepsilon=0.01)$

In the case of small porosity the changes of temperature due to the change of velocity are rather imperceptible (compare Figures 1 and 2). The similar situation takes place for different porosities and small velocity (compare Figures 2, 3 and 5). When porosity $\varepsilon$ is greater than 0.001 and velocity is equal to $u=0.03 \mathrm{~m} / \mathrm{s}$ the maximum temperatures are moved for next moments of times (compare Figures 4 , 6 and 9). Observing the temperature history at the central node of the domain considered (Figures 7 and 8 ) it is visible that in time of heating (10 seconds) the temperature profiles are independent on porosity and velocity, after this time the temperature is changed importantly.

It should be pointed out that for small values of velocity and porosity the results of computations have been compared with the results obtained using the classical 
finite difference method presented in Introduction and the temperatures obtained were practically the same.

\section{Conclusions}

The algorithm based on the finite difference method for numerical solution of the 1D convection-diffusion equation has been proposed. The explicit scheme of FDM has been considered and stability criteria have been formulated. The examples of computations for different values of porosity and velocity have been presented. The drawback of the presented method is the necessity of high-order derivatives approximation.

\section{Acknowledgements}

The paper is a part of the project sponsored by the National Science Centre DEC-2011/01/N/ST6/05231.

\section{References}

[1] Raffray A.R., Pulsifer J., Tillack M.S., Modeling flow and heat transfer through porous media for high heat flux applications, University of California Energy Institute, Berkeley 2000.

[2] Strzelecki T. et al., Modelowanie przepływów przez ośrodki porowate, Dolnośląskie Wydawnictwo Edukacyjne, Wrocław 2008.

[3] Khaled A., Vafai K., The role of porous media in modeling flow and heat transfer in biological tissues, International Journal of Heat and Mass Transfer 2003, 46, 4989-5003.

[4] Kaviany M., Principles of Heat Transfer in Porous Media, 2nd edition, Springer, 1995.

[5] Lewis R.W., Morgan K., Thomas H.R., Seetharamu K.N., The Finite Element Method in Heat Transfer Analysis, John Wiley and Sons, Chichester 1996.

[6] Mochnacki B., Suchy J.S., Numerical Methods in Computations of Foundry Processes, PFTA, Cracow 1995.

[7] Majchrzak E., Mochnacki B., Numerical Methods. Theoretical Bases, Practical Aspects and Algorithms, Publ. of the Silesian University of Technology, Gliwice 2004.

[8] Mochnacki B., Dziewoński M., The evaporation effect in the domain of tissue subjected to a strong external heat source, Scientific Research of the Institute of Mathematics and Computer Science Czestochowa University of Technology 2008, 1(7).

[9] Mochnacki B., Lara-Dziembek S., Weighted residual method as a tool of FDM algorithm construction, Scientific Research of the Institute of Mathematics and Computer Science Czestochowa University of Technology 2010, 1(9), 147-153.

[10] Ciesielski M., Mochnacki B., Szopa R., Numerical modeling of biological tissue heating. Admissible thermal dose, Scientific Research of the Institute of Mathematics and Computer Science Czestochowa University of Technology 2011, 1(10). 